FINAL REPORT

U. S. Department of Energy

\title{
BIODEGRADATION OF CHLORINATED SOLVENTS: REACTIONS NEAR DNAPL AND ENZYME FUNCTION
}

\section{Department of Civil and Environmental Engineering Stanford University, Stanford, CA 94305-4020}

Perry L. McCarty (Lead-PI), (650).723-4131, pmccarty@stanford.edu Alfred M. Spormann (Co-PI), (650).723-3668, spormann@stanford.edu Craig S. Criddle (Co-PI), (650).723-9032, craig.criddle@stanford.edu

Ph.D. candidates: , Min-Ying Chu, Alison Cupples, Michael M. C. Liu Post-Doctoral Scholars: Edward M. Driggers, Q. Shiang Fu, Laurence H. Smith, Yanru Yang

DOE Grant Number DE-FG07-99ER62883

EMSP Project Number 70063

December 11, 2003 


\section{EXECUTIVE SUMMARY}

Chlorinated solvents are among the most widespread groundwater contaminants in the country, contamination which is also among the most difficult and expensive for remediation. These solvents are biodegradable in the absence of oxygen, but this biodegradation requires both a food source for the organisms (electron donor) and the presence of chlorinated solvent biodegrading organisms. These two requirements are present naturally at some contamination sites, leading to natural attenuation of the solvents. If one or both requirements are absent, then engineered bioremediation either through addition of an external electron donor or through bioaugmentation with appropriate microorganisms, or both, may be used for site remediation. The most difficult case for cleanup is when a large residual of undissolved chlorinated solvents are present, residing as dense -non-aqueous-phase- liquid ( DNAPL). A major focus of this study was on the potential for biodegradation of the solvents when present as DNAPL where concentrations are very high and potential for toxicity to microorganisms exist. Another focus was on a better understanding of the biological mechanisms involved in chlorinated solvent biodegradation . These studies were directed towards the chlorinated solvents, trichloroethene (TCE), tetrachloroethene or perchloroethene (PCE), and carbon tetrachloride (CT).

The potential for biodegradation of TCE and PCE DNAPL was clearly demonstrated in this research. From column soil studies and batch studies we found there to be a clear advantage in focusing efforts at bioremediation near the DNAPL. Here, chlorinated solvent concentrations are the highest, both because of more favorable reaction kinetics and because such high solvent concentrations are toxic to microorganisms, such as methanogens, which compete with dehalogenators for the electron donor. Additionally, biodegradation near a PCE DNAPL results in an enhanced dissolution rate for the chlorinated solvent, by factors of three to five times, leading to a more rapid clean-up of the DNAPL zone. The most favored electron donor to add is one which partitions well with the chlorinated solvent or can be concentrated near it. Unfortunately, an ideal electron donor, such as vegetable oil, is difficult to introduce and mix with DNAPL in the ground, doing this properly remains an engineering challenge. Numerical model studies have indicated that several factors may significantly influence the rate and extent of enhancement, including the inhibitory effects of PCE and CDCE, the level of ED concentration, DNAPL configuration, and competition for ED. Such factors need to be considered when contemplating engineered DNAPL bioremediation.

Pseudomonas stuzeri $\mathrm{KC}$ is an organism that transforms $\mathrm{CT}$ to carbon dioxide and chloride without the formation of the hazardous intermediate, chloroform. This is accomplished by production and secretion of a molecule called PDTC. This study was directed towards determining how PDTC works. $\mathrm{Cu}$ (II) at a ratio of 1:1 $\mathrm{Cu}$ to PDTC was found to result in the most rapid $\mathrm{CT}$ transformation, confirming that the PDTC-Cu complex is both a reactant and a catalyst in CT transformation. CT degradation requires that the PDTC be in a reduced form, which is generated by contact with cell components. Fe(II) inhibits CT transformation by PDTC. Studies indicated that this inhibition is enhanced by some compound or factor in the supernatant with molecular weight greater than 10,000 $\mathrm{Da}$. We have made progress in determining what this factor might be, but have not yet been able to identify it. In related studies, we found that CT transformation by another organism, Shewanella oneidensis MR1, also involves an excreted factor, but this factor is different from PDTC and results in chloroform 
transformation as an intermediate. Our studies have indicated that this factor is similar to vitamin $\mathrm{K} 2$, and we have also confirmed that vitamin K2 does transform CT into chloroform.

\section{GOALS AND OBJECTIVES}

The chlorinated solvents, trichloroethene (TCE), tetrachloroethene (PCE), and carbon tetrachloride (CT) have been widely used by industry, the Department of Defense, and the Department of Energy as solvents for cleaning. Through leakage and improper disposal practices, these solvents have become the most frequent groundwater contaminants throughout the country, causing one of the most difficult and costly contamination problems for remediation. Significant groundwater contamination with these solvents exists at DOE facilities such as Savannah River, Hanford, and the Idaho National Engineering Laboratory. For example at operable unit 200-ZP-2 in Hanford, a groundwater volume of over 3.5 square miles in a depth of 270 feet is contaminated with CT at concentrations of up to $3000 \mathrm{ppb}$ (drinking water standard is $5 \mathrm{ppb}$ ). A co-contaminant here is tritium. Most remediation approaches involve the extraction of contaminated groundwater and its cleanup at the surface through expensive physical and/or chemical methods. It is estimated that for the 200 Hanford plume alone, a conventional pump and treat operation has to continue for about 56 years at a total estimated costs exceeding $\$ 50$ million. However, anaerobic biological processes have resulted in the natural destruction (intrinsic bioremediation) of the chlorinated solvents in some cases. Engineered in-situ biodegradative processes offer much promise for complete destruction of the contaminants. In addition at the Hanford facility, surface treatment of water co-contaminated with tritium may require long-term surface storage to allow decay. In situ treatment of chlorinated solvents coupled with natural attenuation of tritium is likely to be a much less expensive alternative. However, the in situ biological processes are not adequately understood at the present and additional basic research on the biological processes involved is essential both for evaluating the true potential for intrinsic bioremediation at some sites and for reliable engineering of the process at others.

The biological anaerobic reductive dehalogenation of chlorinated aliphatic hydrocarbons (CAHs) such as PCE and TCE to cDCE and VC in groundwater was reported in the early 1980s. Further reduction of PCE and its intermediates to the harmless end product ethene was reported in 1989. Several pure cultures of anaerobic bacteria have been found to reductively dehalogenate PCE to CDCE. Rates of reduction of PCE and TCE to $\mathrm{CDCE}$ are high and the need for electron donor addition for the reactions is small. Recently, reductive dehalogenation of PCE and TCE has been observed in the vicinity of DNAPL, raising the opportunity for biodegradation of these chlorinated solvents at the DNAPL source. However, potential toxicity issues, the characteristics of the microorganisms involved, the kinetics of the reactions at high contaminant concentrations, and the molecular basis of the processes involved are not well understood. The aim of research conducted under this grant was to provide a better understanding of the kinetics, chemistry, and microbiology of this process.

The subsequent reduction of $\mathrm{CDCE}$ to $\mathrm{VC}$, and of VC to ethene is much slower than that of PCE dehalogenation, which often results in an accumulation of these compounds. Only recently has a pure culture been reported that is capable of reducing $\mathrm{CDCE}$ to $\mathrm{VC}$ or $\mathrm{VC}$ to ethene. There are numerous reports of such conversions in mixed cultures. This area is where basic research is most in need, and, thus, one subject of this research. 
Carbon tetrachloride is degraded by anaerobic microorganism with the concomitant formation of chloroform as a toxic intermediate which inhibits many microorganisms and eventually the dechlorination process itself. Studies with Pseudomonas stutzeri strain KC, originally isolated in our laboratory, showed that this organism dehalogenates $\mathrm{CT}$ to $\mathrm{CO}_{2}$ under anaerobic condition without the production of $\mathrm{CCl}_{3} \mathrm{H}$. We have used this organism successfully in field-scale bioaumentation studies to remove CT from contaminated aquifers. Bioaugmentation with this organism has great potential for in-situ bioremediation of groundwater, such as at the Hanford site. However, the molecular mechanisms of CT dechlorination by this organism are not yet understood. Our biochemical studies on this problem were directed towards identifying the cellular components involved in this reaction and to identify the rate limiting step.

The specific objectives of this multi-investigator effort were to:

1. Evaluate the potential for chlorinated solvent biodegradation near DNAPLs,

2. Provide a molecular understanding of the biological mechanisms involved,

3. Determine cellular components involved in carbon tetrachloride transformation by Pseudomonas stutzeri strain KC without chloroform formation.

\section{ACCOMPLISHMENTS}

\section{Potential for Biodegradation Near DNAPLs}

A series of batch and column experimental studies have been conducted in order to meet objective one, that is to evaluate the potential for biodegradation of chlorinated solvents at concentrations near saturation. Trichloroethene (TCE) and tetrachloroethene (PCE) were the chlorinated solvents studied. Initially, batch studies were performed to determine the potential of dehalogenating microorganisms to reductively dehalogenate PCE at high concentration, and the effect on the competing ability of other microorganisms, including methanogens and homoacetogens (Yang and McCarty, 2000). Results showed that dehalogenation of PCE can be obtained at super-saturation concentration $(>0.9 \mathrm{mM})$, although long-term contact with PCE DNAPL was inhibitory. TCE was dehalogenated up to $2.26 \mathrm{mM}$. No apparent inhibitory effect on dehalogenation was found with cis-1,2-dichloroethene (cDCE) and ethene at the highest tested levels of $0.66 \mathrm{mM}$ and $1.05 \mathrm{mM}$ respectively. However, such high concentrations of PCE, cDCE and ethene were inhibitory to methanogens and high concentrations of PCE were inhibitory to homoacetogens. Such inhibition is highly beneficial as it greatly diminishes the competition for added electron donor by methanogens and homoacetogens, resulting in more efficient substrate utilization for dehalogenation. A column study was then undertaken in which PCE was added in sufficient amount to fill two percent of the column pore space PCE DNAPL dehalogenation in occurred in the column, and required less than one gram of the electron donor pentanol to dehalogenate one gram of PCE to cDCE. Additionally, DNAPL dissolution rate was significantly enhanced when directly coupled with dehalogenation. The total PCE plus dehalogenation product concentrations equaled over four times the PCE saturation value of $0.9 \mathrm{mM}$. These results demonstrated the significant advantage that can be achieved by carrying out in-situ reductive dehalogenation directly on DNAPL, rather than on the emerging contaminated plume. 
The experimental studies were then expanded to evaluate donor substrates that would offer different remediation strategies for DNAPLs, and to evaluate the toxicity of PCE and intermediate degradation products to different steps in the dehalogenation process (Yang and McCarty, 2002 and 2003). Four parallel continuous-flow columns were constructed to simulate aquifers contaminated with PCE DNAPL ( $2 \%$ residual saturation). One column was used as a negative control to monitor PCE dissolution alone. The other three were pentanol-fed (soluble substrate, fed continuously), oleate-amended (slow hydrogen release following calcium precipitation, placed in column initially), and vegetable oil-amended (mixed with PCE and placed in column initially). During eight months of operation, near saturation concentration of PCE $(0.8$ $\mathrm{mM}$ ) was observed in the effluent of all columns. PCE dehalogenation began after 1-2 months in the three substrate-supplied columns, with cis-1,2-dichloroethene (cDCE) exceeding the effluent PCE concentration. Vinyl chloride (VC) and ethene production were also found in significant amounts $(0.2$ to $0.4 \mathrm{mM})$ although batch studies suggested that significant toxicity from PCE should have occurred. Compared with the control column, the DNAPL dissolution rate was enhanced by about three times when directly coupled with biological degradation. High concentrations of PCE, cDCE, and ethene inhibited methanogens in batch studies, but active methanogenesis occurred in both the pentanol-fed and oleate-amended columns, but not in the vegetable oil-amended column. This suggests that methanogens managed to colonize the column niches where PCE DNAPL was not present. This was confirmed in the pentanol-fed column as methane production decreased significantly when the feeding solution was changed so that it was pre-saturated with PCE. This change was accompanied by a greatly enhanced dechlorination to ethene. These results suggest possible DNAPL remediation strategies $t$ to enhance dehalogenation while controlling competitive methanogenic utilization of donor substrates.

In the above studies, we found that the presence of DNAPL and the competition between dehalogenators and methanogens for electron donor makes it difficult to predict the performance of enhanced dissolution. For example, the transport of compounds may be retarded to different degrees at DNAPL zones. The composition of PCE DNAPL might be significantly changed due to the partitioning back of dehalogenation products. In order to gain a general understanding of the behavior of this complex microbial NAPL system, we have developed numerical models of the process. With a good numerical model, the responses of such a system using different substrate delivery methods can be quickly evaluated; thus, it is possible to optimize the performance of a particular method before conducting actual experiments. One difficulty in modeling the biological reactions under dual transverse mixing is that high reaction rates occur in small regions $(<0.2 \mathrm{~cm})$; therefore, extremely fine grids are required to correctly model the behaviors of the system. Because our goal is to stimulate a high PCE transformation rate exclusively near DNAPLs, detailed modeling is required to understand the extent of bio-enhanced DNAPL destruction and the dynamics of microbial communities around DNAPLs.

Our initial modeling effort was the development of an advection-dispersion-reaction model for a two-dimensional domain, with groundwater flowing over a pool of free-produce (DNAPL) PCE (Chu, Kitanidis, and McCarty, 2003). PCE is converted to cDCE, and toxicity due to PCE or cDCE is neglected. We adopted previously published correlations relating biomass concentrations and hydraulic conductivity, accounting for biofilm growth and plug-like growth. The system of coupled equations is solved numerically. The high biotransformation rate of PCE 
increases the concentration gradient of PCE at the water-DNAPL interface, enhancing dissolution. The higher the electron donor (ED) concentration, the larger the dissolution enhancement. Based on the values of maximum specific rate we used, when the electron donor is unlimited, the active biomass accumulates adjacent to the water-NAPL interface, and microbial reactions can significantly enhance the pool dissolution. The resulting steady-state dissolution rate can be approximated by a half-order solution when zero-order kinetics are suitable for representing the microbial reaction. However, bioclogging may significantly reduce local hydraulic conductivity; thus, it decreases the flow near the water-DNAPL interface, decreasing dissolution. When the ED is the limiting factor, active biomass accumulates away from the interface. This creates a no-flow zone between the active biomass and the interface. The enlargement of the no-flow zone, due to the donor limitation, diminishes the concentration gradient and the flushing around the water-DNAPL interface. Such adverse impacts may significantly decrease the enhancement predicted by models that do not consider the effects of bioclogging.

Through additional modeling efforts, we have found that microbially enhanced dissolution is a complex time-dependent process since the composition and the distribution of DNAPL change over time due to the interactions between dehalogenation reactions and dissolution (Chu, Kitanidis, and McCarty, 2003b and 2003c). This was found through a novel modeling approach that explicitly accounts for the interactions between dissolution, reaction, and hydrodynamics near residual non-aqueous PCE. With the help of this modeling technique, we have found that several factors may significantly influence the rate and extent of enhancement, including the inhibitory effects of PCE and $\mathrm{CDCE}$, the level of ED concentration, DNAPL configuration, and competition for ED. When product inhibition occurs, the enhanced dissolution rate does not increase with the scale of the DNAPL source zone, thereby significantly limiting the extent of enhancement. However, this important issue has not been well addressed. More experimental work is needed for characterizing the dehalogenation and fermentation kinetics at high concentrations of PCE and/or its metabolic products. Experimental studies on CDCE inhibition are underway, but have not been completed sufficiently for this final report.

The simulation results also show how biomass profiles evolve and how dehalogenation activity alters the distributions of various compounds within DNAPL source zones. These effects cannot be captured by a simple mass-transfer macroscopic modeling approach. Although detailed modeling is feasible for small and well-defined domains, how to correctly and efficiently simulate bio-reactive transport through complex stationary DNAPL sources for field applications remains a challenge.

\section{Molecular Mechanisms of Biological Dehalogenation of DNAPL}

\section{1) Engineering improved hydrolytic dehalogenases}

Research under this objective has focused on the natural enzyme haloalkane dehalogenase (DhlA), which is capable of hydrolytically dehalogenating short-chain haloalkanes such as 1,2 dichloroethane (1,2 DCA). Directed evolution experiments with DhlA were conducted with the goal that functional mutants generated can improve our understanding of hydrolytic 
dehalogenation, as well as providing immediate use in applications requiring the dehalogenation of structurally-similar priority pollutants, such as 1,1,1 Trichloroethane (1,1,1 TCA) and 1,1,2 Trichloroethane (1,1,2 TCA).

Initial effort for the directed evolution of DhlA used the error-prone PCR technique, which randomly mutagenizes wildtype $d h l A$ during PCR amplification to generate DhlA variants. Clones generated were screened for enhanced activity against 1,2 DCA, 1,1,1 TCA and 1,1,2 TCA. With an error rate of $0.7 \%$, only 99 of the $1031(9.6 \%)$ mutants generated retained activity against 1,2 DCA. No mutants were found to have activity against 1,1,1 TCA or 1,1,2 TCA.

Later effort shifted to a novel form of family shuffling, which restricts mutations only to those found in other related dehalogenating enzymes. Theoretically the restriction would improve the odds for generating functional enzymes. In practice, this technique does produce more functional mutants at even higher error rates, using 1,2-DCA activity as the benchmark. For comparison, in one such design, with an error rate of $3.4 \%$, about $18 \%$ of the mutants retained activity against 1,2 DCA. Hence, by using this methodology, we are able to access a greater amount of molecular complexity without compromising functional yield.

In our latest effort, we attempted to rapidly identify lead target pollutants that can be transformed by the mutant library generated by our novel family shuffling method. To this end, we purified the enzyme library using affinity chromatography and screened it against 12 environmentally-important halogenated short-chain hydrocarbons, 7 of which are CERCLA priority pollutants, using gas chromatography/mass spectrometry. (Table 1) Comparing to wildtype DhlA, the mutant library showed a shifted activity profile as a population. Significant activity shift was observed for three compounds with improvement relative to wildtype DhlA as indicated: 1,2-dibromo-3-chloropropane (3x improvement), 1,2,3-trichloropropane (8x improvement), and 1,2-dichloropropane (2x improvement), indicating that these are good target compounds for the mutant library. As the screen was done at the population-level, we believe that even higher level of improvement can be expected once the library is sieved for the most improved individual. In addition, this result reflects the functional plasticity of DhlA using this novel mutagenesis methodology.

Table 1. List of halogenated alkanes tested 


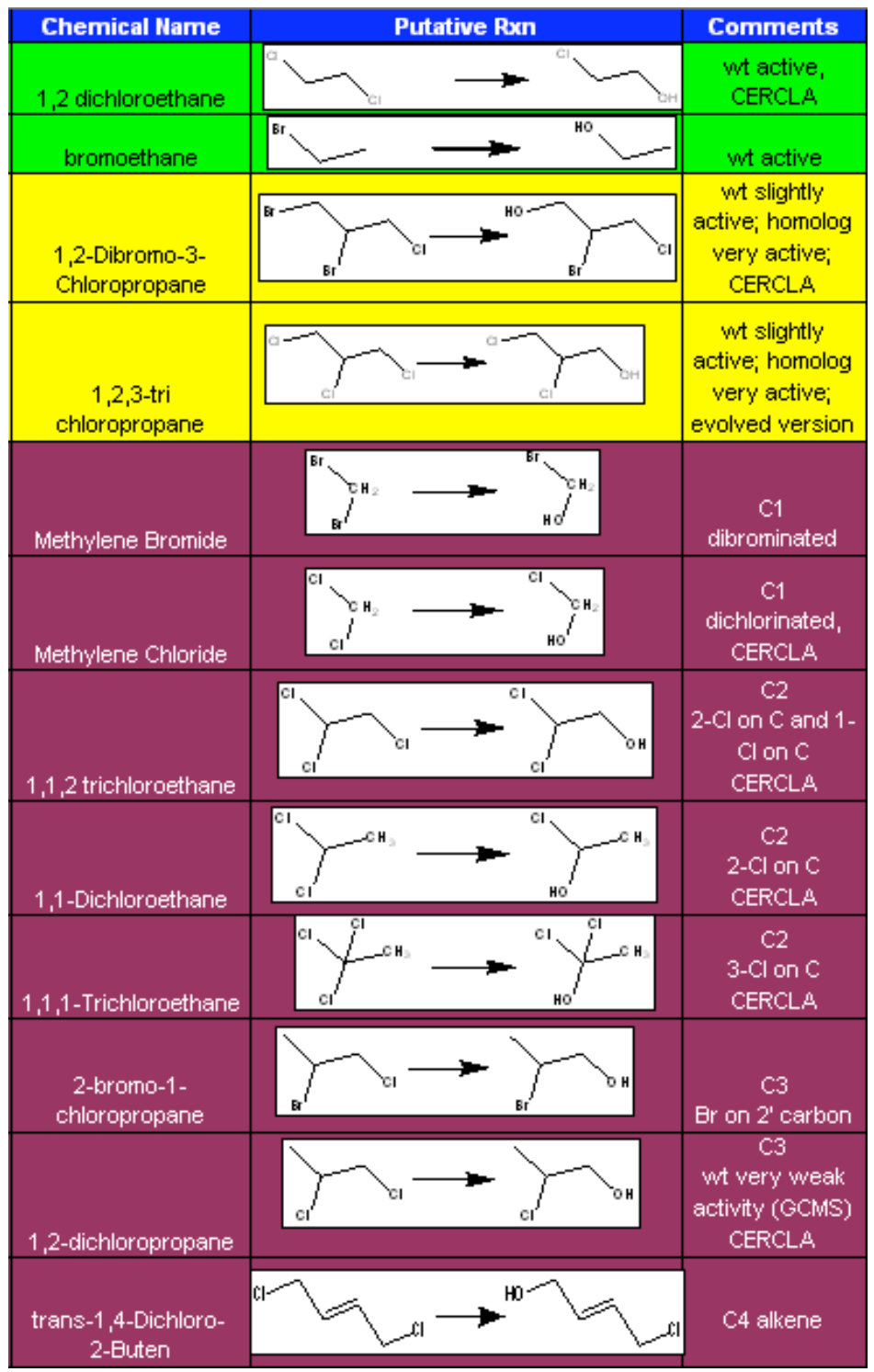

\section{2) Carbon Tetrachloride Transformation}

Studies on Pseudomonas stuzeri KC fall under both objectives 2 and 3. The overall aim in studying strain $\mathrm{KC}$ was to identify components and mechanisms involved in the detoxification of carbon tetrachloride. Microorganisms grown under denitrifying conditions typically produce chloroform, but little chloroform is produced by strain $\mathrm{KC}$, and the major products are instead carbon dioxide and nonvolatile products. This unique transformation is due to the production and secretion of molecule pyridine-2,6 (bis)thiocarboxylate (PDTC) (Lee et al., 1999). When PDTC is chelated to copper, the complex rapidly transforms carbon tetrachloride. Recently, we and collaborators at Michigan State University completed a 5-year full-scale demonstration of strain $\mathrm{KC}$ bioaugmentation for remediation of carbon tetrachloride in an aquifer at Schoolcraft, MI (Dybas et al., 2002). 
In order to quantify the kinetics of carbon tetrachloride transformation by Pseudomonas stutzeri $K C$ and to gain a fuller understanding of the reaction mechanism, we synthesized PDTC in our laboratory. Initially, we evaluated the effects of copper on the transformation in the absence of cells using Ti(II) as the reducing agent. The results showed limited transformation in the presence of PDTC alone. Addition of copper greatly enhanced the rate and extent of carbon tetrachloride removal. A ratio of 1:1 $\mathrm{Cu}$ to PDTC resulted in the fastest transformation with only trace amount of chloroform formation and removal of up to 7.4 mole of carbon tetrachloride. These results confirm that the PDTC-Cu complex is both a reactant and a catalyst in carbon tetrachloride transformation. They also indicate that a catalytic mechanism is operative in reducing environments.

To further understand the mechanisms of this transformation, we developed a highly sensitive and specific detection method for PDTC using triple quad LC/MS/MS with electrospray ionization (ESI). Samples were taken from a growing Pseudomonas stutzeri KC culture and PDTC production was quantified by LC/MS/MS. Pseudomonas stutzeri KC was grown both aerobically and under denitrifying conditions. Parallel experiments were performed to monitor carbon tetrachloride transformation during growth under denitrifying condition. PDTC production was detected under both conditions, but PDTC levels decreased rapidly near the end of the exponential growth period under aerobic conditions. It is not clear whether PDTC was metabolized or decayed into other compounds. When grown with nitrate as the electron acceptor, rapid production of PDTC was observed at the late phase of exponential growth. The production of PDTC was evidently growth-associated as its concentration peaked at the end of the growth phase and stabilized in the stationary phase. This pattern is illustrated in figure 1.

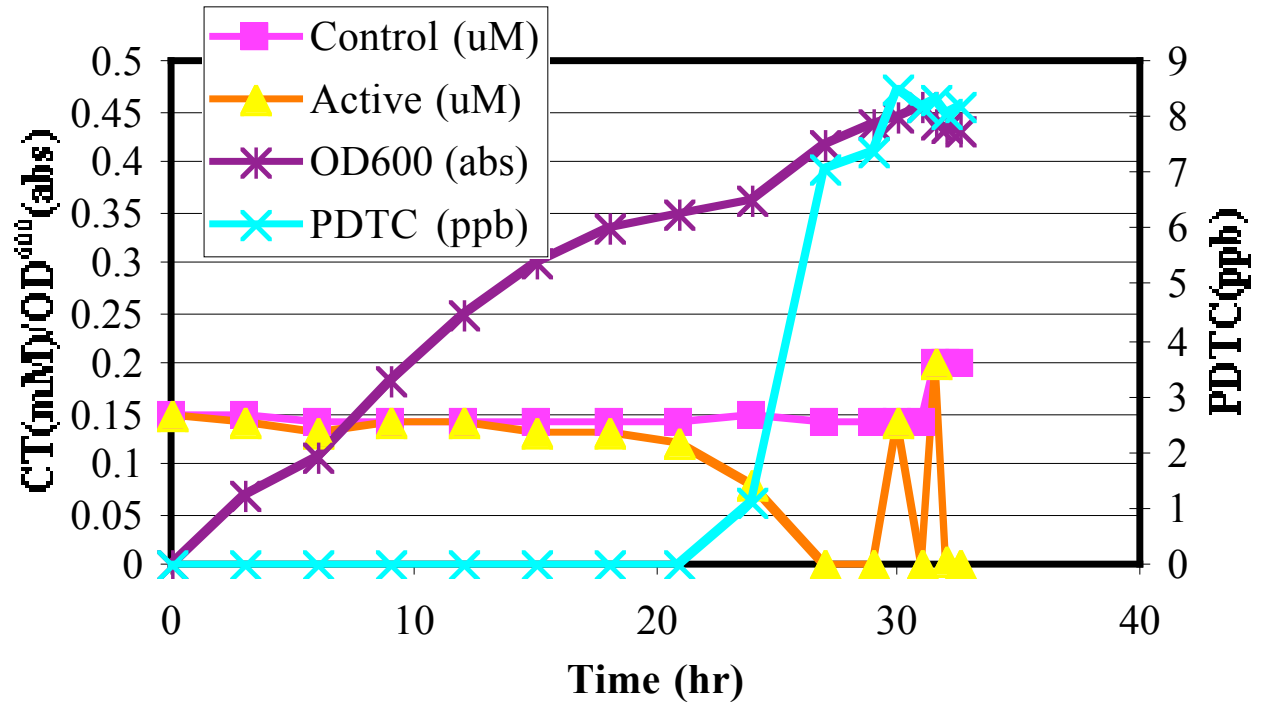

Figure 1. Growth and production of PDTC by Psedudomonas stutzeri KC grown under denitrifying conditions.

As shown in Figure 1, production of PDTC coincided with the onset of carbon tetrachloride transformation. Carbon tetrachloride was added at the initiation of growth. Additional carbon 
tetrachloride spikes were added at concentrations two to three fold higher than the concentration of PDTC produced. These were quickly transformed. This confirms earlier work establishing that the carbon tetrachloride transformation is catalytic and that PDTC-Cu was regenerated for transformation at a rate equal to or greater than the rate of carbon tetrachloride transformation.

To be active for carbon tetrachloride transformation, the PDTC-copper complex must be reduced by cell components. The cell types needed for this reduction step are evidently quite broad, since a wide range of organisms can mediate it, including gram negative bacteria, gram positive bacteria, and eukaryotic cells. Tatara et al. (1995) reported that the only cell type tested that was unable to reduce PDTC were Lactobacillus lacking electron transport chains.

To identify cellular factors responsible for regeneration of PDTC, we used Shewanella oneidensis MR-1 as a model organism for the regeneration step. In order to use this organism for this purpose, we first confirmed that the wild type was able to reduce added PDTC and mediate rapid transformation of carbon tetrachloride. Interestingly, strain MR-1 has the ability to transform carbon tetrachloride by a different mechanism that produces chloroform, but when we added PDTC, the rate of carbon tetrachloride transformation increased significantly, and the only trace amounts of chloroform were produced, demonstrating that PDTC addition successfully altered the mechanism of transformation. With Shewanella an important class of redox-active molecules involved in electron transfer reactions are the menaquinones. Accordingly, we hypothesized that components of the menaquinone biosynthetic pathway might be involved in the reduction of PDTC. To test this hypothesis, we created several MR-1 mutants lacking menaquinone formation capability, then tested these mutants for their rates of carbon tetrachloride transformation and chloroform production. All of the mutants of MR-1 retained the ability to transform carbon tetrachloride at high rates with little chloroform formation. These results indicate that components of the menaquinone biosynthetic pathway are not involved in the established of PDTC for carbon tetrachloride transformation.

Although this line of research did not lead to identification of the cellular components required for reduction of PDTC, it did lead us down an unexpectedly fruitful line of inquiry. In the absence of PDTC addition, certain of the mutants of Shewanella oneidensis MR1 exhibited decreased rates of carbon tetrachloride transformation. Although this pathway is unrelated to our work on PDTC, we followed up this observation by testing the supernatant produced by these mutants, and discovered that wild type Shewanella oneidensis MR1 also secretes a carbon tetrachloridedegrading factor. This factor is unlike PDTC in that it does yield chloroform as a significant reaction product. Although wild type MR-1 produced this factor, some of the mutants did not. LC-MS-MS comparison of the supernatant from the wild type and mutant cultures established that the secreted factor is a simple menaquinone, likely menaquinone-1. Subsequent synthesis and testing of menaquinone- 1 confirmed that this molecule transforms carbon tetrachloride, and produces chloroform as a significant transformation product. This led us to question whether the final product of the menaquinone biosynthetic pathway - menaquinone-7,8 (vitamin K2) - might possess similar activity. We tested this vitamin and found that it did in fact possess such activity. Vitamin K2 is widely distributed in the cellular membranes of microbial populations, and this may help to explain why chloroform formation from carbon tetrachloride is so ubiquitous in nature. To our knowledge, this is the first report of carbon tetrachloride transformation by this family of molecules. 
Returning to our studies of Pseudomonas stutzeri KC, we focused attention on the observations of Dybas et al. (1995). This work demonstrated that addition of micromolar levels of ferric iron to an actively transforming culture inhibited CT transformation, but the level of iron required for inhibition was more than 100-fold higher for washed cells reconstituted with a 10,000-Da supernatant filtrate. From these observations, we surmised that the inhibitory effects of iron were due to a supernatant factor or factors with a molecular weight greater than 10,000 Da. Additional data from proteinase-treated culture dialysate indicated that this factor might consist of one or more proteins.

We hypothesized that the inhibitory secreted factor may be a protein that binds ferric iron and the PDTC-Cu complex or a protein that binds copper and the PDTC-Fe complex. To identify candidate proteins with these properties, we used a combination of immobilized metal affinity capture and Surface Enhanced Laser Desorption Time-of-Flight Mass Spectrometry (SELDI MS). Immobilized metal affinity capture uses an addressable array chip to capture or "dock" one or more proteins based on their affinity for metals attached to the surface - in this case, iron or copper attached to the chip surface by NTA. A protein extract of KC supernatant was then applied to the functionalized chip. Secreted proteins that bind non-specifically with $\mathrm{Fe}$ or $\mathrm{Cu}$ were washed away by a dilute solution of PDTC-Cu solution, leaving proteins with an affinity for both the PDTC-Cu complex and the metal of interest. To size these proteins, we used SELDI MS. A laser pulse was applied to trigger release of adsorbed proteins; their mass was then determined by its time-of-flight in the mass spectrometer.

Using the above strategy, we captured secreted and sized proteins from Pseudomonas stuzeri KC supernatant. The results indicate that several supernatant proteins bind specifically to surfacebound ferric iron and copper. A representative SELDI-MS series of spectra is shown in figure 2. This spectrum shows a Cu-binding protein with a molecular weight of 9600 daltons. The corresponding protein band from a SDS-PAGE gel is shown in Figure 3. The gel results show proteins with sizes that align with those of the SELDI-MS spectra. We are currently isolating and sequencing these proteins. Once the sequences are known, we expect to be able to determine the function of these proteins. This will assist in the development of an accurate conceptual model of the extracellular processes involved in CT transformation by strain $\mathrm{KC}$. 


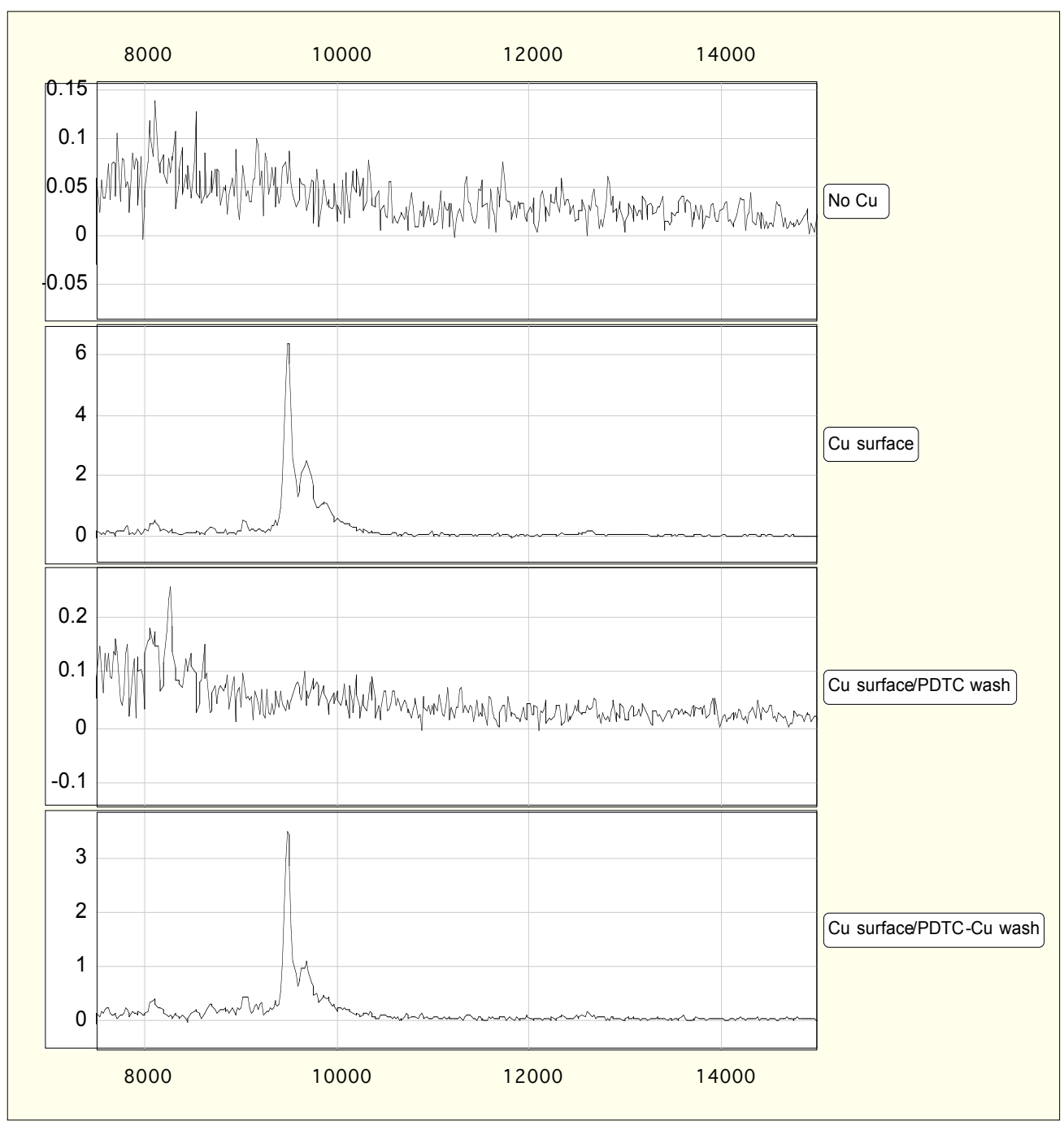

Figure 2. SELDI-MS spectra. Top panel: a chip without $\mathrm{Cu}$ failed to capture proteins. Topmiddle panel: a surface with attached $\mathrm{Cu}$ captured a 9600 dalton protein. Bottom-middle panel: washing of the chip with a PDTC solution removes the $\mathrm{Cu}$-binding protein. Bottom panel: washing of the chip with a solution containing PDTC-Cu complex leaves the protein attached.

This work completed under this funding greatly improves our understanding of the molecular basis of the carbon tetrachloride transformation by strain $\mathrm{KC}$ (and incidentally in strain MR1 as well). We now are preparing to develop a kinetic model capable of representing PDTC production and decay and the kinetics of carbon tetrachloride transformation. This model will enable improved engineering design and enhance the potential for bioremediation of carbon tetrachloride by Pseudomonas stutzeri KC. 


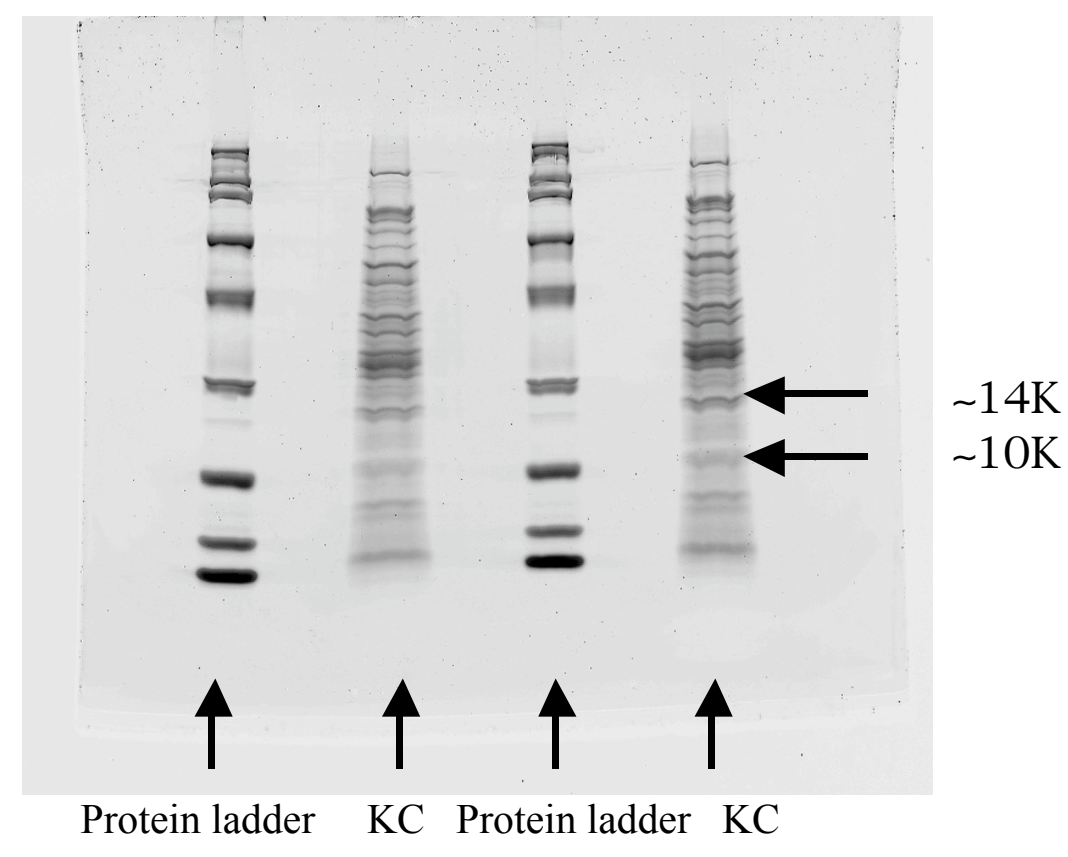

Figure 3. SDS-PAGE gel of protein extract, showing a protein of the same size (9600 daltons) as the one detected by SELDI-MS (see Fig 2).

\section{References}

Dybas, M. J., D. W. Hyndman, R. Heine, K. Linning, J. Tiedje, T. Voice, R. Wallace, D. Wiggert, X. Zhao, L. Dybas and C. S. Criddle, 2002. Development, operation, and long-term performance of a full-scale biocurtain utilizing bioaugmentation. Environ. Science and Technol. $\underline{36}, 3635-3644$

Fu, Q. S., Hobbs S. K., Shi, G., Bednarski, M.D., and Criddle. C. S. 2003. SELDI-MS Characterization of Extracellular Proteins Involved in Carbon Tetrachloride Transformation by Pseudomonas stutzeri KC, Abstract, ASM $103^{\text {rd }}$ General Meeting, Washington DC

Fu, Q. S., Yeung, J. C.H., Rhoads, K, and Criddle, C. S, 2003. Kinetics of Carbon Tetrachloride Transformation by Pseudomonas stutzeri KC, Environmental Science \& Technology (Manuscript in preparation).

Fu, Q. S., Hobbs S. K., Shi, G., Bednarski, M.D., and Criddle. C. S. 2003. Extracellular Proteins Involved in Inhibition of Carbon Tetrachloride Transformation by Pseudomonas stutzeri KC through Binding with $\mathrm{Fe}$ and $\mathrm{Cu}$, Applied and Environmental Microbiology (Manuscript in preparation).

Lee, C.-H., T. A. Lewis, A. Paszczynski, and R. L. Crawford, 1999. Identification of an extracellular agent of carbon tetrachloride dehalogenation from Pseudomonas stutzeri strain KC as pyridine-2, 6-bis(thiocarboxylate).Biochim. Biophys. Res. Commun. 261, 562-566. 
Tatara, G., M. Dybas, and C. S. Criddle, 1995. Biofactor-mediated transformation of carbon tetrachloride by diverse cell types. In: Bioremediation of Chlorinated Solvents. pp. 69-76. Ed: R. E. Hinchee, A. Leeson, and L. Semprini, Bioremediation series 3(4), Battelle Press, Columbus, Ohio.

\section{Acknowledgments}

This material is based upon work supported by the U. S. Department of Energy under DOE Grant Number DE-FG07-99ER62883, and by E. I DuPont de Nemours, Inc. through the U.S. Environmental Protection Agency-sponsored Western Region Hazardous Substance Research Center. Any opinions, findings, and conclusions or recommendations expressed in this material are those of the authors and do not necessarily reflect the views of these organizations.

\section{Project Publications}

Chu, M., Kitanidis P. K. and McCarty P. L. 2003a. Effects of Biomass Accumulation on Biologically Enhanced Dissolution of a PCE Pool: a Numerical Simulation, Journal of Contaminant Hydrology, 65, 75-100.

Chu, M., Kitanidis P. K. and McCarty P. L. 2003b. Large-Time Behavior of GW Pollutant Plumes Subject to Biodegradation at the Fringe: Mathematical Analysis and its Application to a Large-Scale ( $10 \mathrm{~km})$ Field Problem, Eos Trans. AGU, 84(46), Fall Meet. Suppl., Abstract H11D-0886.

Chu, M., Kitanidis P. K. and McCarty P. L. 2003c. Possible Factors Controlling the Effectiveness of Bioenhanced Dissolution of Non-Aqueous Phase Tetrachloroethene, submitted for publication.

Fu, Q. S., Hobbs S. K., Shi, G., Bednarski, M.D., and Criddle. C. S. 2003. SELDI-MS Characterization of Extracellular Proteins Involved in Carbon Tetrachloride Transformation by Pseudomonas stutzeri KC, Abstract, ASM $103^{\text {rd }}$ General Meeting, Washington DC

Fu, Q. S., Yeung, J. C.H., Rhoads, K, and Criddle, C. S, 2003. Kinetics of Carbon Tetrachloride Transformation by Pseudomonas stutzeri KC, Environmental Science \& Technology (Manuscript in preparation).

Fu, Q. S., Hobbs S. K., Shi, G., Bednarski, M.D., and Criddle. C. S. 2003. Extracellular Proteins Involved in Inhibition of Carbon Tetrachloride Transformation by Pseudomonas stutzeri $K C$ through Binding with $\mathrm{Fe}$ and $\mathrm{Cu}$, Applied and Environmental Microbiology (Manuscript in preparation).

Yang, Y., and McCarty, P. L. 2003. Response to Comment on "Comparison of Donor Substrates for Biologically Enhanced Tetrachloroethene (PCE) DNAPL Dissolution”, Environmental Science \& Technology, 37(11), 2620-2621. 
Yang, Y., and McCarty, P. L. 2002. Comparison of Donor Substrates for Biologically Enhanced Tetrachloroethene (PCE) DNAPL Dissolution, Environmental Science \& Technology, 36(15), 3400-3404.

McCarty, P. L. 2001. Strategies for In-Situ Bioremediation of Chlorinated Solvent Contaminated Groundwater, Groundwater Quality 2001, University of Sheffield, Sheffield, UK, 12-15 (2001).

Yang, Y. and McCarty, P. L. 2000. Biologically Enhanced Dissolution of Tetrachloroethene DNAPL, Environmental Science \& Technology, 34(14), 2979-2984. 